\title{
Cell-cycle length of medial ganglionic eminence progenitors contributes to interneuron fate
}

\section{Dear Editor,}

GABAergic interneurons contribute to cortical function by regulating the balance of activity. Malfunction of inhibitory interneurons has been associated with neuropsychiatric disorders (Hashimoto et al. 2003; Marín 2012). The most striking feature of GABAergic interneurons is their diversity of cell types. Although fate-mapping studies have demonstrated the presence of spatially and temporally distinct progenitor domains for interneuron subclasses within the embryonic ventral telencephalon (Butt et al. 2005; Inan et al. 2012), the mechanisms of interneuron subtype-determination during development remain poorly understood.

Considerable evidence supports the idea that cell fate determination throughout neurogenesis is intimately linked with cell-cycle length (Ohnuma and Harris 2003; Pilaz et al. 2016). In fact, disruption of normal cell-cycle length is known to alter neurogenesis (Hardwick et al. 2015; Boyd et al. 2015). However, due to technical limitations, we still lack detailed information about the specific impact of cell-cycle length on cell fate determination in vivo. An important unanswered question is whether there is a direct relationship between cell-cycle length of neural progenitors and interneuron fate determination.

In this study, we developed a triple thymidine analog labeling method to label progenitors undergoing short cycles (PSC) or progenitors undergoing long cycles (PLC) in the medial ganglionic eminence (MGE) in vivo (Fig. $1 \mathrm{~A}-\mathrm{C}$ ). In brief, we carried out an initial injection of IdU followed $2 \mathrm{~h}$ later by an injection of BrdU at embryonic day 13.5 (E13.5). Cells labeled by IdU, but not BrdU (IdU $\left.{ }^{+} \mathrm{BrdU}^{-}\right)$had left $\mathrm{S}$ phase. After various intervals $(4,9,12$ and $14 \mathrm{~h}$, defined as $\Delta \mathrm{T})$, we injected EdU to label cells that re-entered $\mathrm{S}$ phase $\left(\right.$ IdU $\left.^{+} \mathrm{BrdU}^{-} \mathrm{EdU}^{+}\right)$(Fig. 1A). Using this labeling method, the cell-cycle lengths $\left(T_{C}\right)$ of $\mathrm{IdU}^{+} \mathrm{BrdU}^{-} \mathrm{EdU}^{+}$cells can be roughly calculated for different $\Delta \mathrm{Ts}$ (Fig. 1B). We found that the percentage of $\mathrm{IdU}^{+} \mathrm{BrdU}^{-} \mathrm{EdU}^{+}$cells in IdU $\mathrm{BrdU}^{+}$cells in $\Delta T=9 \mathrm{~h}$ group was around three times higher than in the other $\Delta \mathrm{T}$ groups at E13.5 (Figs. $1 \mathrm{D}$ and $\mathrm{S} 1 \mathrm{~A}-\mathrm{H}$; Table S1); whereas, the peak of $\Delta T$ at E15.5 was $12 \mathrm{~h}$ (Figs. $1 \mathrm{E}$ and S1I-P; Table S1), longer than at E13.5, suggesting that the average cell-cycle length of MGE progenitors gradually increases during embryonic development. To further characterize the variability of cell-cycle length, we used timelapse microscopy to monitor cell-cycle progression in cultured MGE cells at E13.5 (Fig. S2A-C). Histogram analysis showed that the distribution of cell-cycle lengths of MGE progenitors occupied a wide range, between 8 and $22 \mathrm{~h}$ (Fig. S2D). The average cell-cycle length of cultured MGE cells at E13.5 was $12.20 \pm 0.20 \mathrm{~h}$, which was significantly shorter than at E15.5 (20.11 $\pm 1.16 \mathrm{~h})$ (Fig. S2E; Table S1). In addition, we found that only $24.0 \%$ of lineages showed relatively stable cell-cycle length in 3-round divisions (relative variation less than $10 \%$ ) (Fig. S2F and S2G). These results suggest that cell-cycle length in individual lineages of MGE progenitors over multiple divisions exhibits remarkable heterogeneity and instability.

To determine whether $\mathrm{IdU}^{+} \mathrm{BrdU}^{-} \mathrm{EdU}^{+}$cells do indeed undergo division, and if so, how many times they divide, we monitored the lineages of cultured MGE cells at E13.5, and sequentially applied IdU, BrdU and EdU into the culture medium to mimic the triple-labeling method in vivo (Fig. $1 \mathrm{~F}$ and $1 \mathrm{G}$ ). Indeed, for each $\Delta \mathrm{T}, 100 \%$ of $\mathrm{IdU}^{+} \mathrm{BrdU}^{-} \mathrm{EdU}^{+}$cells divided at least once during observation (Figs. 1G-I and $\mathrm{S} 2 \mathrm{H}$; Table S1). However, in $\Delta \mathrm{T}=14 \mathrm{~h}$ group, $34.05 \% \pm$ $5.66 \%$ of $\mathrm{IdU}^{+} \mathrm{BrdU}^{-} \mathrm{EdU}^{+}$cells divided twice (Fig. $1 \mathrm{H}$ and 1 ; Table S1), suggesting that about one-third of $\mathrm{IdU}^{+} \mathrm{Br}$ $\mathrm{dU}^{-} E \mathrm{dU}^{+}$cells in $\Delta \mathrm{T}=14 \mathrm{~h}$ group were progenitors undergoing short cycles. During measuring the cell-cycle length of MGE progenitors in the different $\Delta \mathrm{T}$ groups, we found that the ranges of cell-cycle length for $10 \%-90 \%$ progenitors in the $\Delta \mathrm{T}=4 \mathrm{~h}, \Delta \mathrm{T}=9 \mathrm{~h}$ and $\Delta \mathrm{T}=12 \mathrm{~h}$ groups were $6-12 \mathrm{~h}$, 11-15 $\mathrm{h}$ and 13-18 $\mathrm{h}$, respectively (Fig. S2I and S2J; Table S1). Taken together, these results indicate that, for $\Delta T$ $\leq 12 \mathrm{~h}$, the triple-labeling method can specifically label MGE progenitors with different cell-cycle lengths at E13.5, and the progenitors labeled in the $\Delta \mathrm{T}=4 \mathrm{~h}$ and $\Delta \mathrm{T}=12 \mathrm{~h}$ have distinct cell-cycle lengths.

As mentioned above, PSC and PLC in MGE at E13.5 can be individually labeled in vivo by the triple-labeling method when $\Delta T=4 \mathrm{~h}$ and $\Delta T=12 \mathrm{~h}$. We divided the MGE in coronal brain slices into six $30^{\circ}$ sectors (Fig. $1 \mathrm{~J}$ ), the density 

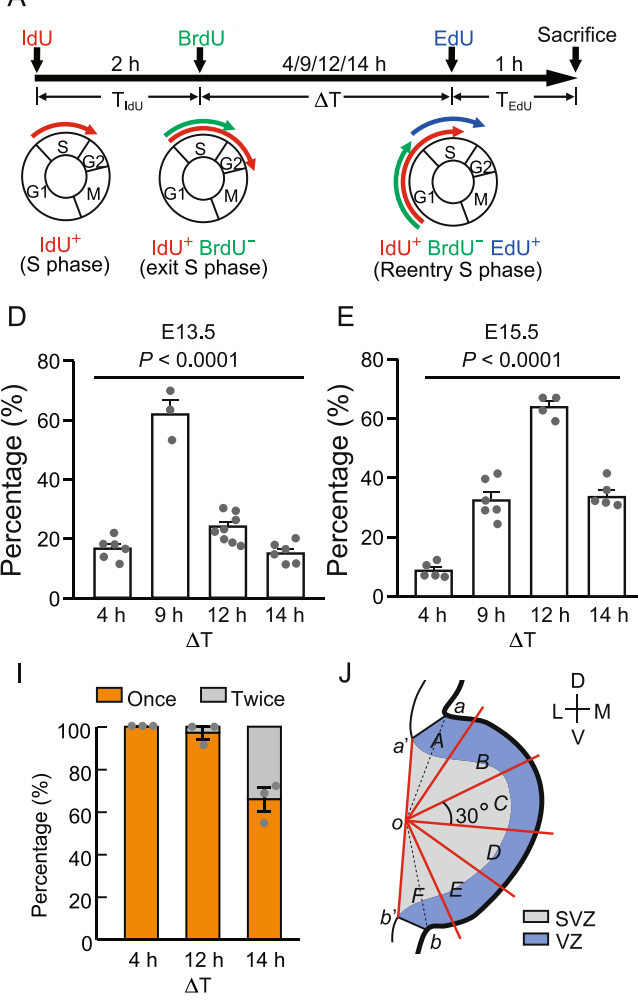

E
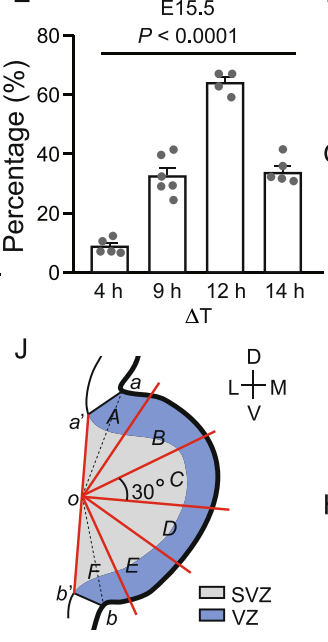

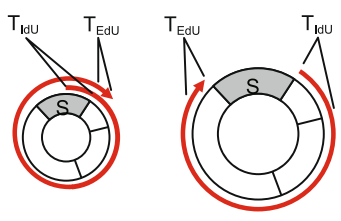

$\Delta T<T_{\mathrm{C}}<\mathrm{T}_{\text {ldU }}+\Delta \mathrm{T}+\mathrm{T}_{\mathrm{EdU}}+\mathrm{T}_{\mathrm{S}}$

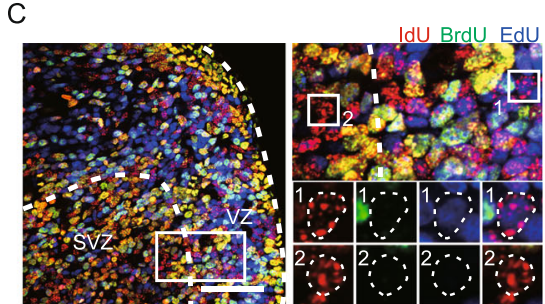

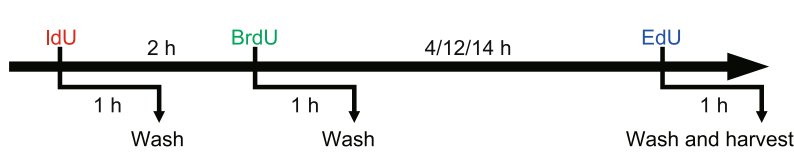
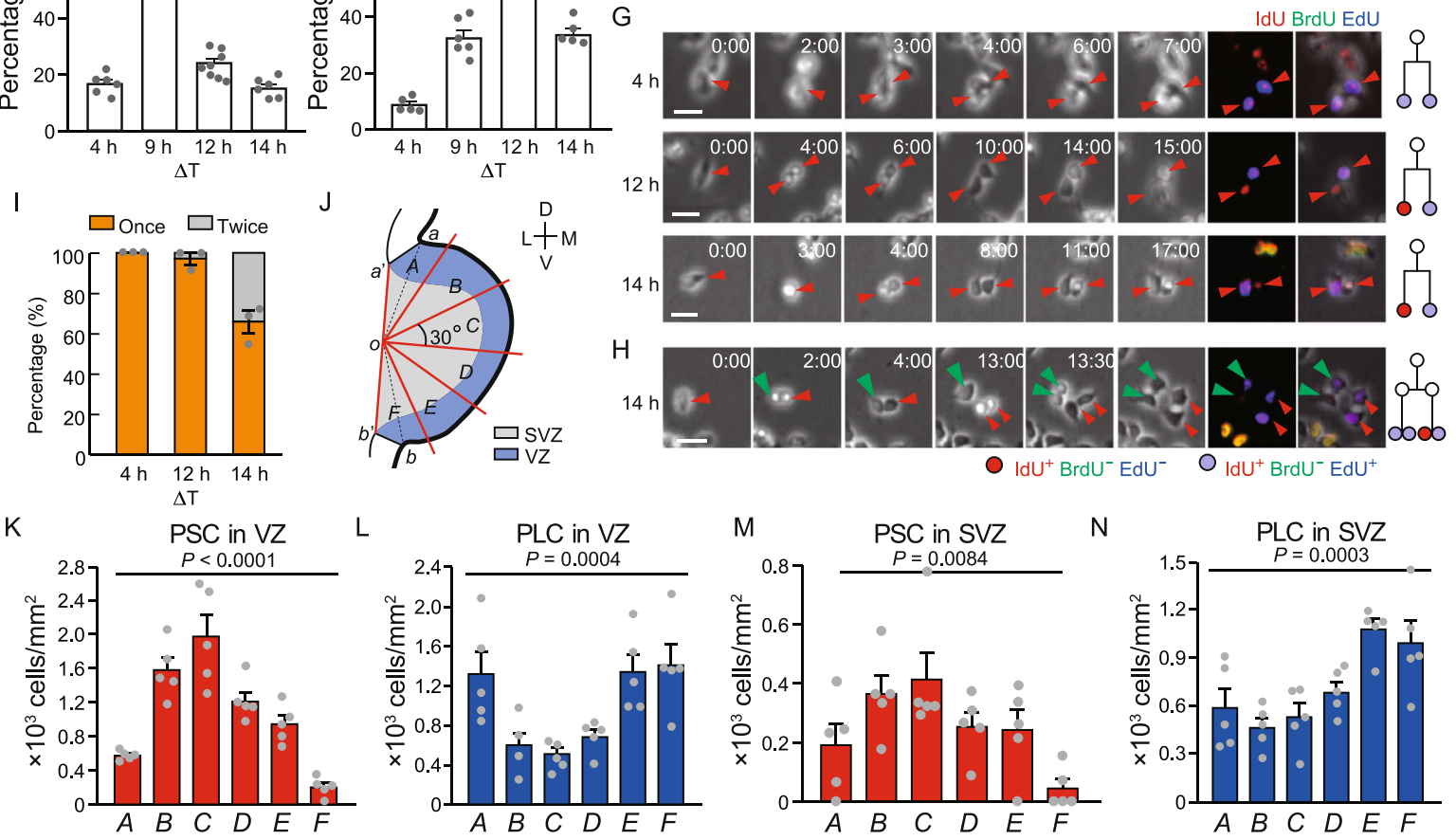

O

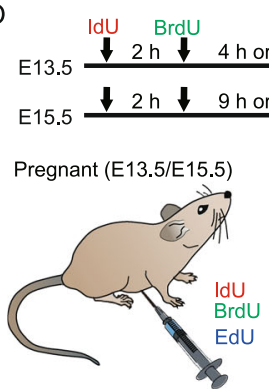

$\mathrm{P}$

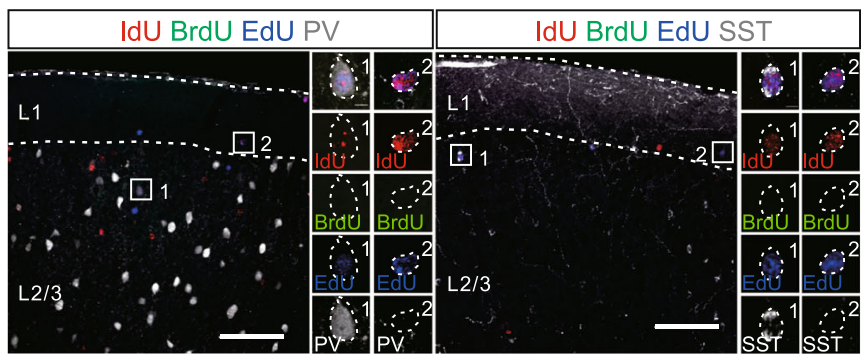

Q
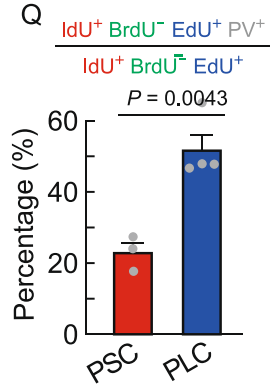

$S$

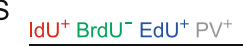
$\mathrm{IdU}^{+} \mathrm{BrdU}^{-} \mathrm{EdU}^{+}$

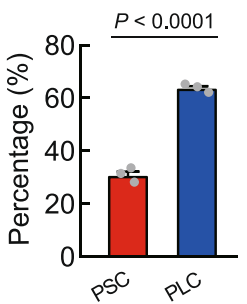

$\mathrm{R}$ $\frac{\mathrm{IdU}^{+} \mathrm{BrdU}^{-} \mathrm{EdU}^{+}}{P=0.0000}$

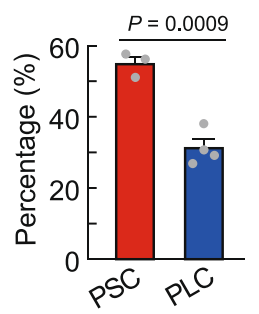

T

$\underline{\mathrm{ddU}^{+} \mathrm{BrdU}^{-} \mathrm{EdU}^{+} \mathrm{SST}^{+}}$

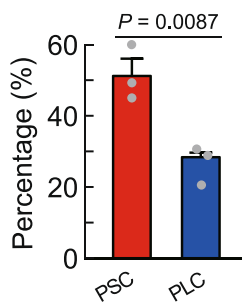


4 Figure 1. MGE progenitor cells with different cell-cycle lengths are biased toward generating specific cortical interneuron subgroups. (A) Experimental outline. Pregnant mice at E13.5 and E15.5 received an injection of IdU and $2 \mathrm{~h}$ later BrdU, followed by an injection of EdU at various intervals $(4,9,12$ or $14 \mathrm{~h} ; \Delta \mathrm{T})$. Brains were collected for analysis $1 \mathrm{~h}$ after EdU injection. (B) The estimated range of cell-cycle lengths for a specific $\Delta T$. (C) Representative image of thymidine analogtreated MGE at E13.5 $(\Delta T=4 \mathrm{~h})$, magnified images on the right (rectangular region). Cell 1 is $\mathrm{IdU}^{+} \mathrm{BrdU}^{-} \mathrm{EdU}^{+}$, and cell 2 is $\mathrm{IdU}^{+} \mathrm{BrdU}^{-} \mathrm{EdU}^{-}$. Scale bar, $50 \mu \mathrm{m}$. (D and E) The percentage of $\mathrm{IdU}^{+} \mathrm{BrdU}^{-} \mathrm{EdU}^{+}$cells in IdU $\mathrm{BrdU}^{-}$cells in E13.5 and E15.5 MGE under different $\Delta T$ conditions. ( $F$ ) Experimental outline in cultured MGE cells. ( $G$ and $H$ ) Representative time-lapse and immunostained images of cultured MGE cells for different $\Delta T$ conditions ( $4 \mathrm{~h}, 12 \mathrm{~h}, 14 \mathrm{~h}$ ). Scale bar, $20 \mu \mathrm{m}$. (I) Bar plot showing frequency of division for Id $\mathrm{I}^{+} \mathrm{BrdU}^{-} \mathrm{EdU}^{+}$cells during recording under different $\Delta T$ conditions. Data are presented as mean \pm SEM. (J) Schema depicting the partitioning of the MGE into six sectors (See Supplementary Materials). (K-N) The density of PSC and PLC in A-F VZ sectors and A-F SVZ sectors. (O) Experimental outline showing thymidine analog injections and MGE cell transplantation and the anatomical structures from which the embryonic tissues were harvested for transplantation. $(P)$ Representative images showing thymidine analogs co-labeling with PV (left panel, $\Delta \mathrm{T}=4 \mathrm{~h}$ ) or SST (right panel, $\Delta \mathrm{T}=4 \mathrm{~h}$ ) in the cortex of P30 host mice. Cell 1 and 2 in the left panel are $\mathrm{IdU}^{+} \mathrm{BrdU}^{-} \mathrm{EdU}^{+} \mathrm{PV}^{+}$and $\mathrm{IdU}^{+} \mathrm{BrdU}^{-} \mathrm{EdU}^{+} \mathrm{PV}^{-}$, respectively. Cell 1 and 2 in the right panel are $\mathrm{IdU}^{+} \mathrm{BrdU}^{-} \mathrm{E}-$ $\mathrm{dU}^{+} \mathrm{SST}^{+}$and $\mathrm{IdU}^{+} \mathrm{BrdU}^{-} \mathrm{EdU}^{+} \mathrm{SST}^{-}$, respectively. Scale bar, $100 \mu \mathrm{m}$. (Q and R) Quantification of the percentage of PSC and PLC that were positive for PV or SST when transplanted at E13.5. (S and T) Quantification of the percentage of PSC and PLC that were positive for PV or SST when transplanted at E15.5. Data are presented as mean \pm SEM.

of IdU $\mathrm{BrdU}^{-}$cells exhibited no significant difference across the six regions under $\Delta \mathrm{T}=4$ and $12 \mathrm{~h}$ conditions (Fig. S3AD; Table S1). Interestingly, the density of PSC in the middle of the ventricular zone (VZ) was significantly higher than in the dorsal and ventral VZ (Fig. 1K; Table S1). In contrast, the density of PLC in the middle of the VZ was dramatically lower than in the dorsal and ventral VZ (Fig. 1L; Table S1). In the subventricular zone (SVZ), we observed a similar bias in PSC and PLC distribution as in the VZ (Fig. $1 \mathrm{M}$ and $1 \mathrm{~N}$; Table S1). These results suggested that PSC mainly locate in the middle MGE (mMGE), whereas PLC predominantly distribute in the dorsal (dMGE) and ventral MGE (vMGE). We also found that the density of PSC in the caudal MGE was significantly higher than in the rostral and central MGE; whereas the density of PLC in the rostral MGE was significantly higher than in the central and caudal MGE (Fig. S3I$\mathrm{K}$; Table S1). Several studies have identified two main classes of neural progenitors in the MGE: apical progenitors
(APs) which divide along the ventricular surface and basal progenitors (BPS) which divide within the SVZ (Arai et al. 2011). To examine the distribution of PSC and PLC across these two subtypes of neural progenitors, we labeled APs and BPs with Olig2 and cyclin-D2 (CCND2). We found that the percentage of $\mathrm{IdU}^{+} \mathrm{BrdU}^{-} \mathrm{EdU}^{+} \mathrm{Olig} 2^{+} \mathrm{VZ}$ cells in $\mathrm{IdU}^{+} \mathrm{BrdU}^{-} \mathrm{EdU}^{+} \mathrm{VZ}$ cells was comparable between PSC and PLC (Fig. S3L and S3M; Table S1). However, there was a higher percentage of IdU ${ }^{+} \mathrm{BrdU}^{-} \mathrm{EdU}^{+} \mathrm{CCND}^{+} \mathrm{SVZ}$ cells in the PLC than in the PSC (Fig. S3N and S3O; Table S1), suggesting that there are more PLC than PSC in MGE BPs at E13.5.

In this study, the terms PSC and PLC are based on a single cycle. Several lines of evidence suggest that transplanted MGE cells undergo their final division and exit the cell cycle after transplantation into the host brain (Valcanis and Tan 2003; De la Cruz et al. 2011). To study the relationship between the 'single round' cell-cycle length of MGE progenitors and interneuron subgroup fate determination, we transplanted MGE cells from triple thymidine analog-injected mice at E13.5 and E15.5 into the neocortex of postnatal day 1-2 (P1-P2) hosts (Fig. 1O). PSC and PLC at E13.5 were labeled under $\Delta T=4 \mathrm{~h}$ and $\Delta \mathrm{T}=12 \mathrm{~h}$ conditions; at $\mathrm{E} 15.5$, PSC and PLC were labeled under $\Delta \mathrm{T}=9 \mathrm{~h}$ and $\Delta \mathrm{T}=14 \mathrm{~h}$ conditions. In the host cortex, PSC- and PLC-derived interneurons were identified as IdU $\mathrm{BrdU}^{-} \mathrm{EdU}^{+}$. To examine the differentiation of MGE progenitors, we injected thymidine analogs into green fluorescent protein positive $\left(\mathrm{GFP}^{+}\right)$donor mice. Thymidine analog-labeled cells displayed a characteristic interneuron morphology, indicating that transplanted thymidine analog-labeled cells can develop into mature interneurons in the host neocortex (Fig. S4A-D). Co-labeling of $\mathrm{IdU}^{+} \mathrm{BrdU}^{-} \mathrm{EdU}^{+}$with PV or SST markers (labeling two major non-overlapping interneurons subgroups derived from MGE) was then quantified in host cortical sections at P30 (Fig. 1P). We observed that PLC were two times more likely to give rise to $\mathrm{PV}^{+}$interneurons compared to $\mathrm{PSC}$; in marked contrast, PSC produced roughly twice the number of $\mathrm{SST}^{+}$ interneurons compared to PLC at E13.5. (Fig. $1 Q$ and $1 R$; Table S1). Similar results were obtained in transplantations from E15.5 MGE progenitors into P1 neocortex (Fig. 1S and $1 \mathrm{~T}$; Table S1). Together, these results suggest that PSC and $\mathrm{PLC}$ are biased toward generating $\mathrm{SST}^{+}$and $\mathrm{PV}^{+}$interneuron subgroups, respectively.

We then asked what factors regulate cell-cycle length of MGE progenitors and contribute to cell-cycle heterogeneity. Protein $\mathrm{p} 27^{\mathrm{Kip} 1}$ (also called cyclin-dependent kinase inhibitor $1 \mathrm{~B}, \mathrm{CDKN1B}$ ) is an inhibitor of cyclin-dependent kinase involved in the regulation of the cell cycle (Polyak et al. 1994), and widely expressed in MGE progenitors. Thus, we speculate that p27 might be a key factor that regulates the cell-cycle length of MGE progenitors. To investigate this, we generated $\mathrm{Nkx} 2.1-\mathrm{Cre}^{+/-} ; p 27^{f / f l}$ mice (p27 knockout line, p27-cKO) and $\mathrm{Nk} \times 2.1-\mathrm{Cre}^{-/-} ; \mathrm{p} 27^{\mathrm{fllfl}}$ littermates (wild-type line, $p 27-W T$ ) to conditionally deleted the $p 27^{K i p 1}$ gene in the MGE (Fig. 2A). p27 expression in MGE was detected by 

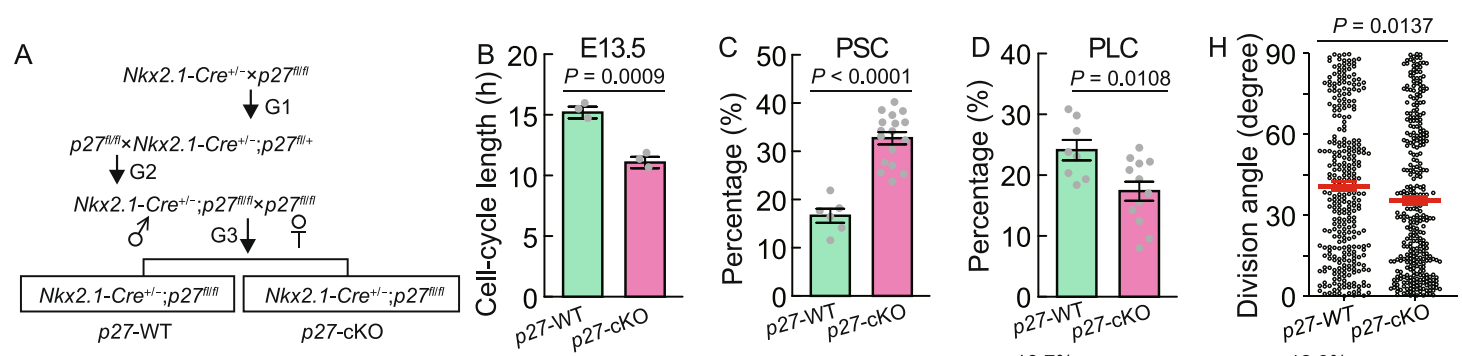

E

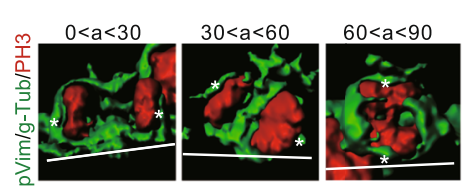

$1 \quad$ p27-WT-PV 2 27-CKO-PV 27 27-WT-SST p27-CKO-SST
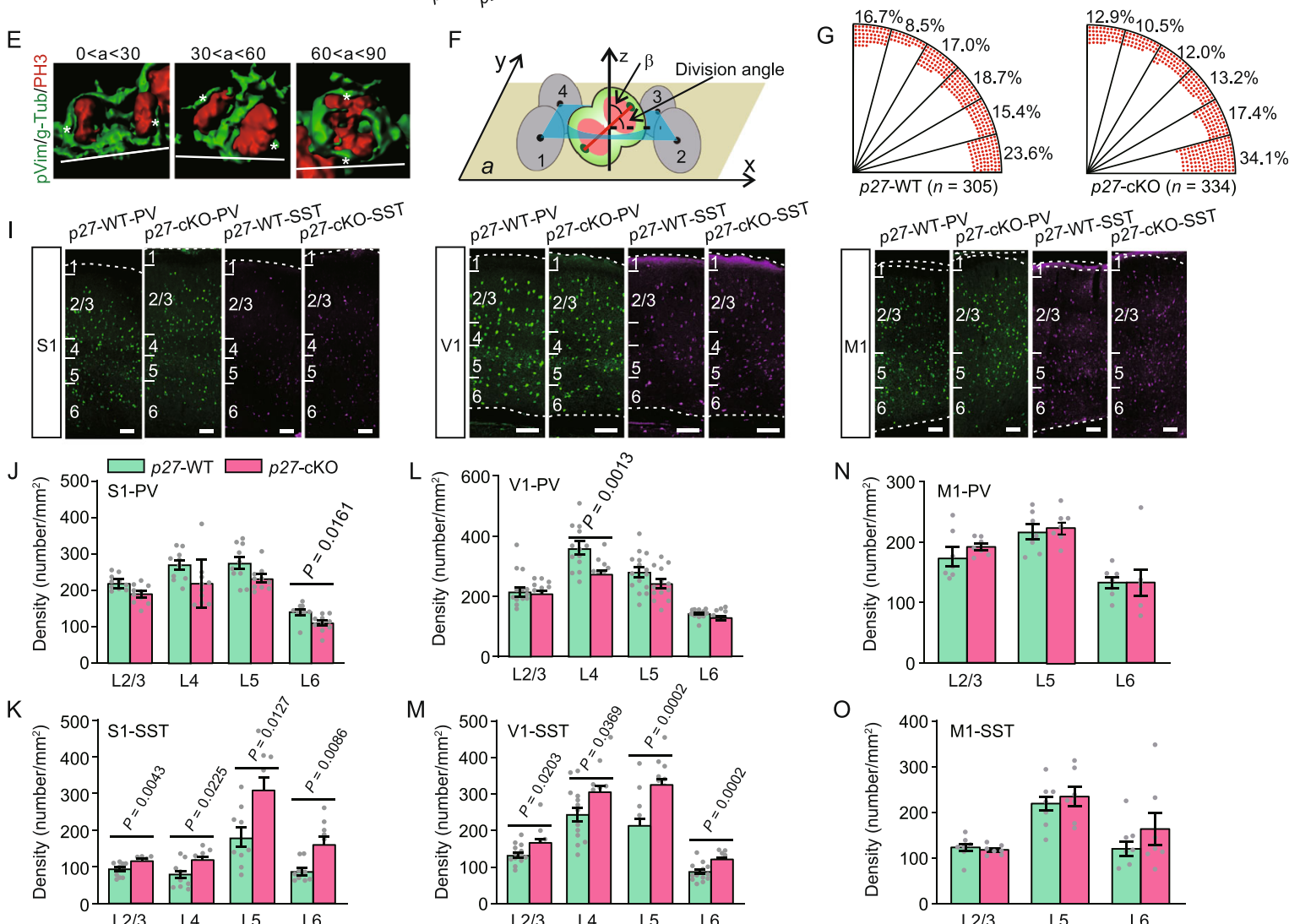

$\mathrm{N}$

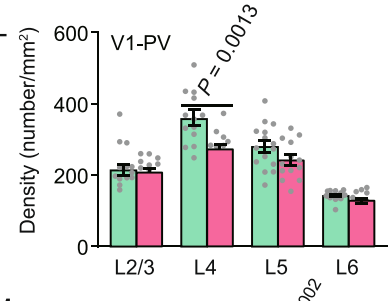

"'
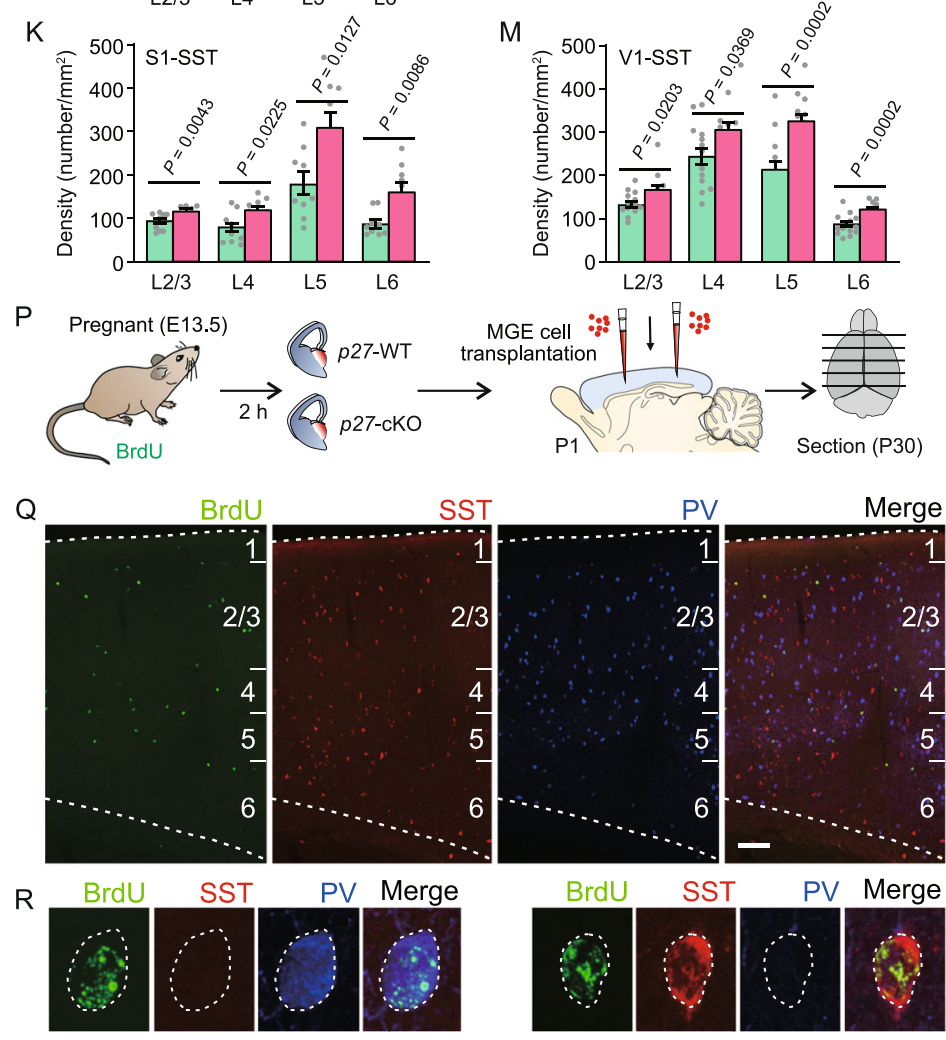

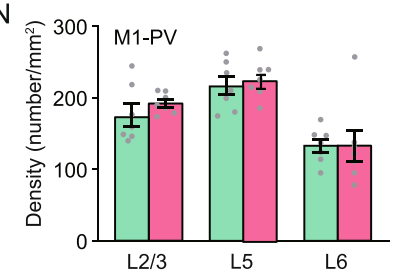

0

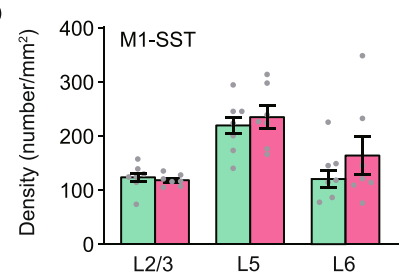

S PV and SST T PV-INs

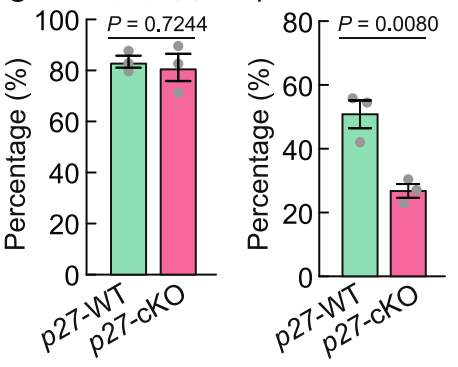

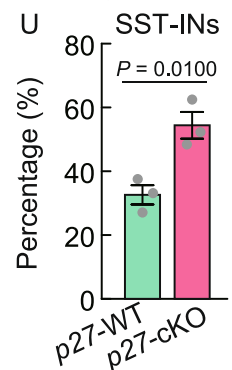


Figure 2. Deletion of p27 shorten the cell-cycle length of MGE progenitors and affect cortical $\mathrm{PV}^{+}$and $\mathrm{SST}^{+}$interneuron production. (A) Illustration of mouse breeding strategy. (B) The average cell-cycle length of E13.5 MGE cells of p27-WT or p27-cKO mice. (C and D) Quantification of the percentage of PSC and PLC in E13.5 MGE of $p 27-W T$ mice and $p 27-c K O$ mice. (E) 3D reconstructions of single-dividing RGCs in the MGE illustrating the three classes of division based on the mitotic cleavage orientation. MGE ventricular surface is at the bottom (white line). Asterisks indicate the centrosomes. (F) Model for the calculation of cleavage plane angle of dividing RGCs in the MGE. Mitotic DNA is in red; the centrosome is in dark green. Cells 1-4 are located at the ventricular surface and adjacent to the targeted cell. Plane $a$, the best-fitting plane for the centers of cells $1-4 . \beta$ is the angle between the vector connecting the two centrosomes and the normal vector ( $z$ axis) of the plane a. (G) Distribution of the division angles of MGE progenitor cells in p27-WT mice and p27-cKO mice. $(\mathrm{H})$ Statistical analysis of division angles between $p 27-\mathrm{WT}$ and $p 27-\mathrm{cKO}$ mice. Data are presented as mean \pm SEM. (I) Representative images of PV and SST immunostaining in S1, V1 and M1 from p27-WT and $p 27-c K O$ mice at P30. Scale bar, $100 \mu \mathrm{m}$. ( $\mathrm{J}$ and K) Quantification of the density of $\mathrm{PV}^{+}$and $\mathrm{SST}^{+}$interneurons in S1 of $p 27-W T$ and $p 27-c K O$ mice. Each data point represents one brain section. ( $\mathrm{L}$ and $\mathrm{M}$ ) Quantification of the density of $\mathrm{PV}^{+}$ and $\mathrm{SST}^{+}$interneurons in V1 of $p 27-\mathrm{WT}$ and $p 27-\mathrm{cKO}$ mice. Each data point represents one brain section. ( $\mathrm{N}$ and $\mathrm{O}$ ) Quantification of the density of $\mathrm{PV}^{+}$and $\mathrm{SST}^{+}$interneurons in M1 of $p 27-W T$ and $p 27-c K O$ mice. Each data point represents one brain section. Data are presented as mean \pm SEM. (P) Experimental outline showing transplantation of MGE cells from E13.5 BrdU-injected embryos into the neocortex of $\mathrm{P} 1$ neonates. ( $Q$ ) Representative images showing BrdU co-labeling with PV or SST in the cortex of P30 host mice. Scale bar, $100 \mu \mathrm{m}$. (R) Two sample cells showing BrdU co-labeling with PV or SST, respectively. (S) Quantification of the percentage represents the sum of $\mathrm{PV}^{+} \mathrm{BrdU}^{+}$and $\mathrm{SST}^{+} \mathrm{BrdU}^{+}$cells in $\mathrm{BrdU}^{+}$ cells in $p 27-W T$ and $p 27-c K O$ group. (T and $U$ ) Quantification of the percentage of transplanted cells from $p 27-\mathrm{WT}$ and $p 27-\mathrm{cKO}$ mice that were positive for PV or SST. Data are presented as mean \pm SEM.

in situ hybridization at E13.5. We found that $p 27$ was only removed in MGE progenitors of $p 27$-cKO mouse line and not in other domains (Fig. S5A). Using dual thymidine analog labeling approaches (BrdU/EdU) in combination with Ki67 labeling at E13.5, we observed that the average $T_{C}$ of MGE progenitors in $p 27-c K O$ at E13.5 was significantly shorter than in p27-WT (Fig. 2B; Table S2). Moreover, we found that the percentage of PSC at E13.5 in p27-cKO mice was around two times higher than in p27-WT mice (Fig. 2C; Table S2). However, the percentage of PLC in p27-cKO mice was significantly lower than in p27-WT mice (Fig. 2D;
Table S2). These data indicate that deletion of p27 can shorten the cell-cycle length of neural progenitors in the MGE. Accumulated evidence indicates that cleavage plane orientation governs the fate of neural stem cell progeny (Chenn and McConnell 1995; Haydar et al. 2003). To examine whether cell-cycle length regulates mitotic spindle orientation of embryonic MGE progenitors, we calculate the cleavage plane angle of APs (Fig. $2 E$ and $2 F$ ). We observed that the fraction of horizontally oriented mitotic spindles (between $0^{\circ}$ and $15^{\circ}$ ) in p27-cKO mice were strongly increased as compared with p27-WT mice (Fig. 2G). Correspondingly, there was significantly difference in division angles between p27-WT and p27-cKO mice (Fig. 2H; Table S2). These data indicate that deletion of $p 27$ can affect mitotic cleavage orientation of neural progenitors in the MGE.

We then addressed a critical question: is p27 expression necessary for the balance of cortical interneuron subtypes derived from MGE? PV and SST immunoreactivity was analyzed in the primary somatosensory cortex (S1), primary visual cortex (V1), primary motor cortex (M1) and striatum of p27-WT and p27-cKO mice at P30 (Figs. 2I and S5B-G). We found that the conditional deletion of $p 27$ in MGE progenitors increased the number of cortical $\mathrm{SST}^{+}$interneurons, but had little effect on $\mathrm{PV}^{+}$interneuron numbers (Figs. 2J-O, S5D and S5G; Table S2). To analyze apoptosis effects of p27 deletion, we labeled apoptotic cells with cleaved caspase-3 at postnatal day 7 (Fig. $\mathrm{S} 5 \mathrm{H}$ ), the peak of endogenous interneuron cell death in neocortex (Southwell et al. 2012). We found that $p 27-c K O$ mice exhibit a similar level of interneuron loss in neocortex as p27-WT mice at P7 (Fig. S5I; Table S2). Finally, we queried whether these phenotypes were evident specifically in MGE progenitors at E13.5. We transplanted MGE cells from E13.5 BrdU-injected embryos into the neocortex of P1 neonates (Fig. 2P-R). Interestingly, the percentage of BrdU-expressing transplanted cells that co-labeled with PV or SST did not differ between p27-WT and p27-cKO groups (Fig. 2S; Table S2), suggesting that deletion of p27 does not alter MGE progenitors to primarily differentiate into $\mathrm{PV}^{+}$and $\mathrm{SST}^{+}$ interneurons. We did not observe PV and SST double positive cells $\left(\mathrm{PV}^{+} / \mathrm{SST}^{+}\right.$cells) in transplanted cells. Remarkably, we found that the percentage of $\mathrm{PV}^{+} \mathrm{BrdU}^{+}$cells in $\mathrm{BrdU}^{+}$cells was reduced by half in the p27-cKO group compared to p27-WT group (Fig. 2T; Table S2). Conversely, there was about 2-fold higher co-localization of SST with BrdU in the p27-cKO group compared to p27-WT group (Fig. 2U; Table S2). Of note, compared to transplantation (Fig. 2P-U), there isn't much of a change in $\mathrm{PV}^{+}$interneuron numbers in the cortex of p27-cKO mice at P30 (Fig. 2J and $2 \mathrm{~L})$. We speculate that the temporal-spatial change of cellcycle length of MGE progenitors induced by p27-cKO may result in differences in $\mathrm{PV}^{+}$interneuron phenotype in transplantation versus the p27 mutant. Taken together, these genetic studies demonstrate that p27 as a key factor 
regulates cortical interneuron fate determination by adjusting cell-cycle length of MGE progenitors.

In summary, we utilized a triple thymidine analog labeling method for the first time to label individual progenitors undergoing short or long cycles in vivo. Our data provide direct evidence in support of a causal link between the cellcycle length of MGE progenitors and cell fate determination of their progeny. We found that PSC mainly give rise to $\mathrm{SST}^{+}$ interneurons, whereas PLC generates predominantly $\mathrm{PV}^{+}$ interneurons. Moreover, we also identified p27 as a key molecule in regulating cortical interneuron fate determination by adjusting cell-cycle length of MGE progenitors. However, the complete molecular mechanisms are still unclear. Future effort is necessary to understand other molecular and cellular mechanisms by which the cell-cycle length of MGE progenitors influences the fate determination of cortical interneuron subtypes. Elucidating these mechanisms will be crucial for fundamentally understanding interneuron subtype-determination, and for ultimately treating the numerous disorders associated with interneuron dysfunction.

\section{FOOTNOTES}

We thank Mi Zhou for her technical support with the time-lapse video recording, and members of the Y.-C.Y. laboratory for their valuable input. This work was supported by grants from the Shanghai Natural Science Foundation (20ZR1403800) to Y.F. The project was also supported by the Natural Science Foundation of China (31930044, 31725012), the Foundation of Shanghai Municipal Education Commission (2019-01-07-00-07-E00062), the Collaborative Innovation Program of Shanghai Municipal Health Commission (2020CXJQ01), the Shanghai Municipal Science and Technology Major Project (No.2018SHZDZX01) and ZJLab to Y.-C.Y. The authors declare no competing interests. All institutional and national guidelines for the care and use of laboratory animals were followed. All data generated or analyzed during this study are included in this published article.

N.Z., M.W., Y.F., and Y.-C.Y. conceived the project and designed the experiments; N.Z., M.W., Y.F. and performed research, D.S. performed some immunohistochemistry; N.Z., M.W., Y.F. and D.S. analyzed data; Y.-C.Y., N.Z., M.W. and Y.F. wrote the manuscript with comments from all the other authors.

\author{
Ni Zong, Min Wang, Yinghui Fu, Dan Shen, Yong-Chun Yu \\ Jing'an District Central Hospital of Shanghai, State Key Laboratory \\ of Medical Neurobiology and MOE Frontiers Center for Brain \\ Science, Institutes of Brain Science, Fudan University, Shanghai \\ 200032, China \\ $\triangle$ Correspondence: ycyu@fudan.edu.cn (Y.-C. Yu) \\ Accepted March 22, 2021
}

\section{OPEN ACCESS}

This article is licensed under a Creative Commons Attribution 4.0 International License, which permits use, sharing, adaptation, distribution and reproduction in any medium or format, as long as you give appropriate credit to the original author(s) and the source, provide a link to the Creative Commons licence, and indicate if changes were made. The images or other third party material in this article are included in the article's Creative Commons licence, unless indicated otherwise in a credit line to the material. If material is not included in the article's Creative Commons licence and your intended use is not permitted by statutory regulation or exceeds the permitted use, you will need to obtain permission directly from the copyright holder. To view a copy of this licence, visit http:// creativecommons.org/licenses/by/4.0/.

\section{REFERENCES}

Arai Y, Pulvers JN, Haffner C, Schilling B, Nüsslein I, Calegari F, Huttner WB (2011) Neural stem and progenitor cells shorten S-phase on commitment to neuron production. Nat Commun 2:154

Boyd JL, Skove SL, Rouanet JP, Pilaz LJ, Bepler T, Gordân R, Wray GA, Silver DL (2015) Human-Chimpanzee differences in a FZD8 enhancer alter cell-cycle dynamics in the developing neocortex. Curr Biol 25:772-779

Butt SJ, Fuccillo M, Nery S, Noctor S, Kriegstein A, Corbin JG, Fishell G (2005) The temporal and spatial origins of cortical interneurons predict their physiological subtype. Neuron 48:591604

Chenn A, McConnell SK (1995) Cleavage orientation and the asymmetric inheritance of Notch1 immunoreactivity in mammalian neurogenesis. Cell 82:631-641

De la Cruz E, Zhao M, Guo L, Ma H, Anderson SA, Schwartz TH (2011) Interneuron progenitors attenuate the power of acute focal ictal discharges. Neurotherapeutics 8:763-773

Hardwick LJA, Ali FR, Azzarelli R, Philpott A (2015) Cell cycle regulation of proliferation versus differentiation in the central nervous system. Cell Tissue Res 359:187-200

Hashimoto T, Volk DW, Eggan SM et al (2003) Gene expression deficits in a subclass of GABA neurons in the prefrontal cortex of subjects with schizophrenia. J Neurosci 23:6315-6326

Haydar TF, Ang E, Rakic P (2003) Mitotic spindle rotation and mode of cell division in the developing telencephalon. Proc Natl Acad Sci USA 100:2890-2895

Inan M, Welagen J, Anderson SA (2012) Spatial and temporal bias in the mitotic origins of somatostatin- and parvalbumin-expressing interneuron subgroups and the chandelier subtype in the medial ganglionic eminence. Cereb Cortex 22:820-827

Marín O (2012) Interneuron dysfunction in psychiatric disorders. Nat Rev Neurosci 13:107-120 
Ohnuma S, Harris WA (2003) Neurogenesis and the cell cycle. Neuron 40:199-208

Pilaz L-J, McMahon JJ, Miller EE et al (2016) Prolonged mitosis of neural progenitors alters cell fate in the developing brain. Neuron 89:83-99

Polyak K, Kato JY, Solomon MJ et al (1994) p27Kip1, a cyclin-Cdk inhibitor, links transforming growth factor-beta and contact inhibition to cell cycle arrest. Genes Dev 8:9-22
Southwell DG, Paredes MF, Galvao RP et al (2012) Intrinsically determined cell death of developing cortical interneurons. Nature 491:109-113

Valcanis H, Tan S-S (2003) Layer specification of transplanted interneurons in developing mouse neocortex. J Neurosci 23:5113-5122

Supplementary Information The online version contains supplementary material available at https://doi.org/10.1007/s13238-02100851-w.

$\mathrm{Ni}$ Zong, Min Wang, Yinghui Fu have contributed equally to this work. 